\title{
Molecular Mechanism for the Interactions of Hofmeister Cations with Macromolecules in Aqueous Solution
}

Ellen E. Bruce, Halil I. Okur, Sina Stegmaier, Chad I. Drexler, Bradley A. Rogers, Nico F. A. van der Vegt,* Sylvie Roke,* and Paul S. Cremer*

Cite This: J. Am. Chem. Soc. 2020, 142, 19094-19100

ACCESS | Lلll Metrics \& More | 回 Article Recommendations | sl Supporting Information

ABSTRACT: Ion identity and concentration influence the solubility of macromolecules. To date, substantial effort has been focused on obtaining a molecular level understanding of specific effects for anions. By contrast, the role of cations has received significantly less attention and the underlying mechanisms by which cations interact with macromolecules remain more elusive. To address this issue, the solubility of poly( $\mathrm{N}$-isopropylacrylamide), a thermoresponsive polymer with an amide moiety on its side chain, was studied in aqueous solutions with a series of nine different cation chloride salts as a function of salt concentration. Phase transition temperature measurements were

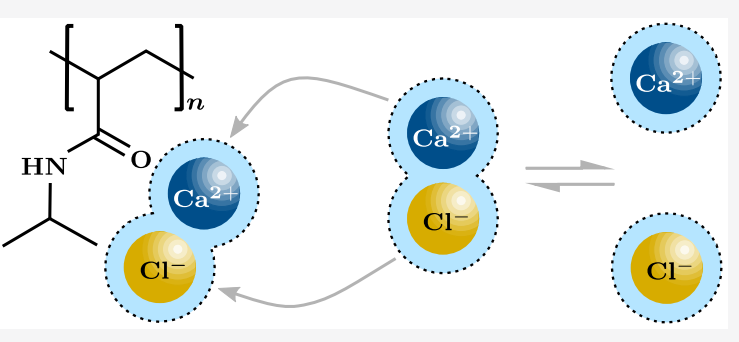
correlated to molecular dynamics simulations. The results showed that although all cations were on average depleted from the macromolecule/water interface, more strongly hydrated cations were able to locally accumulate around the amide oxygen. These weakly favorable interactions helped to partially offset the salting-out effect. Moreover, the cations approached the interface together with chloride counterions in solvent-shared ion pairs. Because ion pairing was concentration-dependent, the mitigation of the dominant salting-out effect became greater as the salt concentration was increased. Weakly hydrated cations showed less propensity for ion pairing and weaker affinity for the amide oxygen. As such, there was substantially less mitigation of the net salting-out effect for these ions, even at high salt concentrations.

\section{INTRODUCTION}

It has been known for more than 130 years that salt ions modulate the physical properties of macromolecules in aqueous solutions. ${ }^{1-33}$ For proteins, a typical consensus cation series for salting-out behavior is as follows: ${ }^{34} \mathrm{Mg}^{2+}<\mathrm{Ca}^{2+}<$ $\mathrm{Li}^{+}<\mathrm{Na}^{+}<\mathrm{K}^{+}<\mathrm{Rb}^{+}<\mathrm{Cs}^{+}<\mathrm{NH}_{4}{ }^{+}<\mathrm{N}\left(\mathrm{CH}_{3}\right)_{4}{ }^{+}$. This series is often dominated by cation interactions with negatively charged carboxylate moieties from aspartate and glutamate residues. ${ }^{15,35,36}$ Although the above series is widely regarded as a direct cationic Hofmeister series, it is neither generic nor well understood. In the absence of negatively charged functional groups, cation interactions with the polypeptide backbone are generally quite weak. As such, cations have often been treated as passive counterions that balance the charge in aqueous solution. A few studies have, however, explored the interactions of cations with the amide oxygen. ${ }^{37,38}$ The results show that strongly hydrated cations (e.g., $\mathrm{Mg}^{2+}, \mathrm{Ca}^{2+}$, and $\mathrm{Li}^{+}$) interact weakly with the amide oxygen, while weakly hydrated cations (e.g., $\mathrm{Na}^{+}$and $\mathrm{K}^{+}$) are depleted from the amide group. $^{25,37,38}$

Herein, we report the lower critical solution temperature (LCST) for poly( $N$-isopropylacrylamide) (PNIPAM). This thermoresponsive polymer exhibits cation specific salting-out behavior with a series of chloride salts $\left(\mathrm{Mg}^{2+}, \mathrm{Ca}^{2+}, \mathrm{Sr}^{2+}, \mathrm{Li}^{+}\right.$, $\mathrm{NH}_{4}^{+}, \mathrm{Na}^{+}, \mathrm{K}^{+}, \mathrm{Rb}^{+}$, and $\left.\mathrm{Cs}^{+}\right)$. In previous studies, the LCST behavior for the anionic Hofmeister series was found to correlate with changes in surface tension at the air/water interface per mole of added salt, $\mathrm{d} \gamma_{\mathrm{s}} / \mathrm{d} c_{\mathrm{s}}$, when the counterion was $\mathrm{Na}^{+}$(where $\gamma_{\mathrm{s}}$ denotes the surface tension and $c_{\mathrm{s}}$ stands for the concentration of salt). ${ }^{8}$ As will be shown herein, this trend is not followed for cation chloride salts. Instead, a cation specific salting-in contribution was observed for all cations and especially for salt solutions of the most strongly hydrated cations. By employing all-atom molecular dynamics (MD) simulations, we elucidated this partial salting-in contribution to macromolecule solubility at the molecular level.

Figure 1 schematically depicts the molecular level mechanism for the cationic Hofmeister series with PNIPAM. Despite net ion depletion from the macromolecule/water interface, local accumulation of strongly hydrated cations occurs at the amide oxygen. Such weak local ion partitioning originates from weak, but energetically favorable interactions between the amide oxygen and the cations. The partitioning of cations to

Received: July 5, 2020

Published: October 30, 2020 


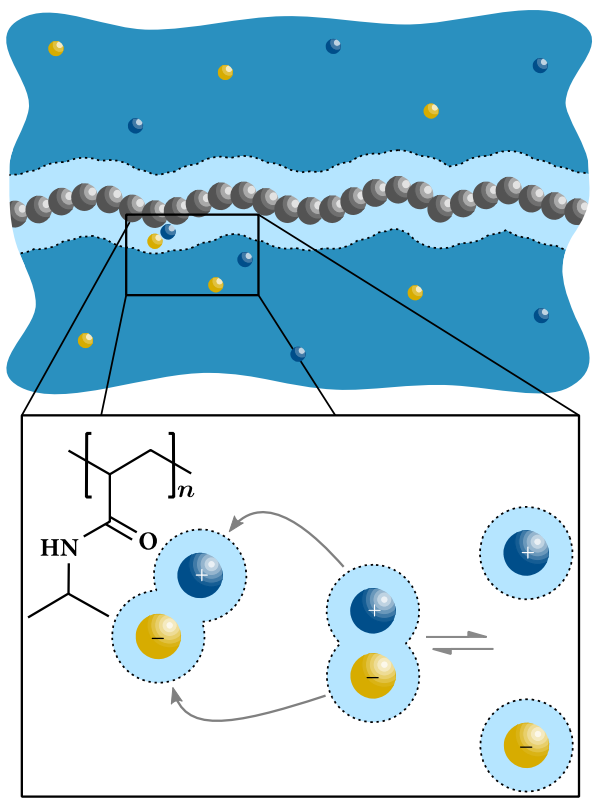

Figure 1. Schematic illustration of the molecular mechanism for the cationic Hofmeister series. (top) The light blue region adjacent to the polymer represents hydration water. (bottom) The partitioning of ions in the form of solvent-shared ion pairs to the vicinity of the amide moiety on a PNIPAM side chain is depicted. The polymer size in the experiments described herein is on the order $n \approx 160$. It should be noted that cations are net depleted from the polymer/water interface. However, when they do come to the interface, they do so together with chloride anions as depicted by the arrows. The relative sizes of the polymer, ions, and hydration shells are not drawn to scale.

the vicinity of the macromolecule is aided by solvent-shared ion pair formation between cations and chloride counterions at the polymer interface. The preference for ion pairing is greater for strongly hydrated cations (e.g., $\mathrm{Ca}^{2+}$ and $\mathrm{Li}^{+}$) than for more weakly hydrated ones (e.g., $\mathrm{Na}^{+}$and $\mathrm{Cs}^{+}$). Because the effect is dependent on the simultaneous presence of both the anion and cation at the polymer surface, it occurs more readily at higher salt concentrations.

\section{RESULTS}

Phase Transition Measurements. Figures $2 \mathrm{a}$ and $2 \mathrm{~b}$ plot the phase transition temperature of PNIPAM as a function of chloride salt concentration for monovalent and divalent cations, respectively. In the absence of salt, the phase transition occurred at $32.1{ }^{\circ} \mathrm{C}$. In the presence of salt, the LCST decreased with increasing salt concentration. Moreover, the various salts influenced the solubility of the macromolecule in a cation specific manner. The salting-out order at a concentration of $1 \mathrm{M}$ was $\mathrm{Li}^{+} \sim \mathrm{NH}_{4}{ }^{+}<\mathrm{Rb}^{+} \sim \mathrm{K}^{+} \sim \mathrm{Cs}^{+} \sim \mathrm{Na}^{+}$for the monovalent cations and $\mathrm{Mg}^{2+}<\mathrm{Ca}^{2+}<\mathrm{Sr}^{2+}$ for the divalent cations. As can be seen by comparing the magnitudes on the $y$ axes of the two plots, the monovalent and divalent salts decreased the LCST to roughly the same extent. This result is surprising because the salts of divalent cations might be expected to be twice as effective at salting-out PNIPAM on a per molar basis. Indeed, the salting-out order for the anionic Hofmeister series correlates strongly with $\mathrm{d} \gamma_{\mathrm{s}} / \mathrm{d} c_{\mathrm{s}}$ (see Table S2) $)^{8,10}$ and the value of $\mathrm{d} \gamma_{\mathrm{s}} / \mathrm{d} c_{\mathrm{s}}$ is primarily dependent on the identity of the anion. In fact, the chloride salts of divalent cations show $\mathrm{d} \gamma_{\mathrm{s}} / \mathrm{d} c_{\mathrm{s}}$ values for the air/water interface that are
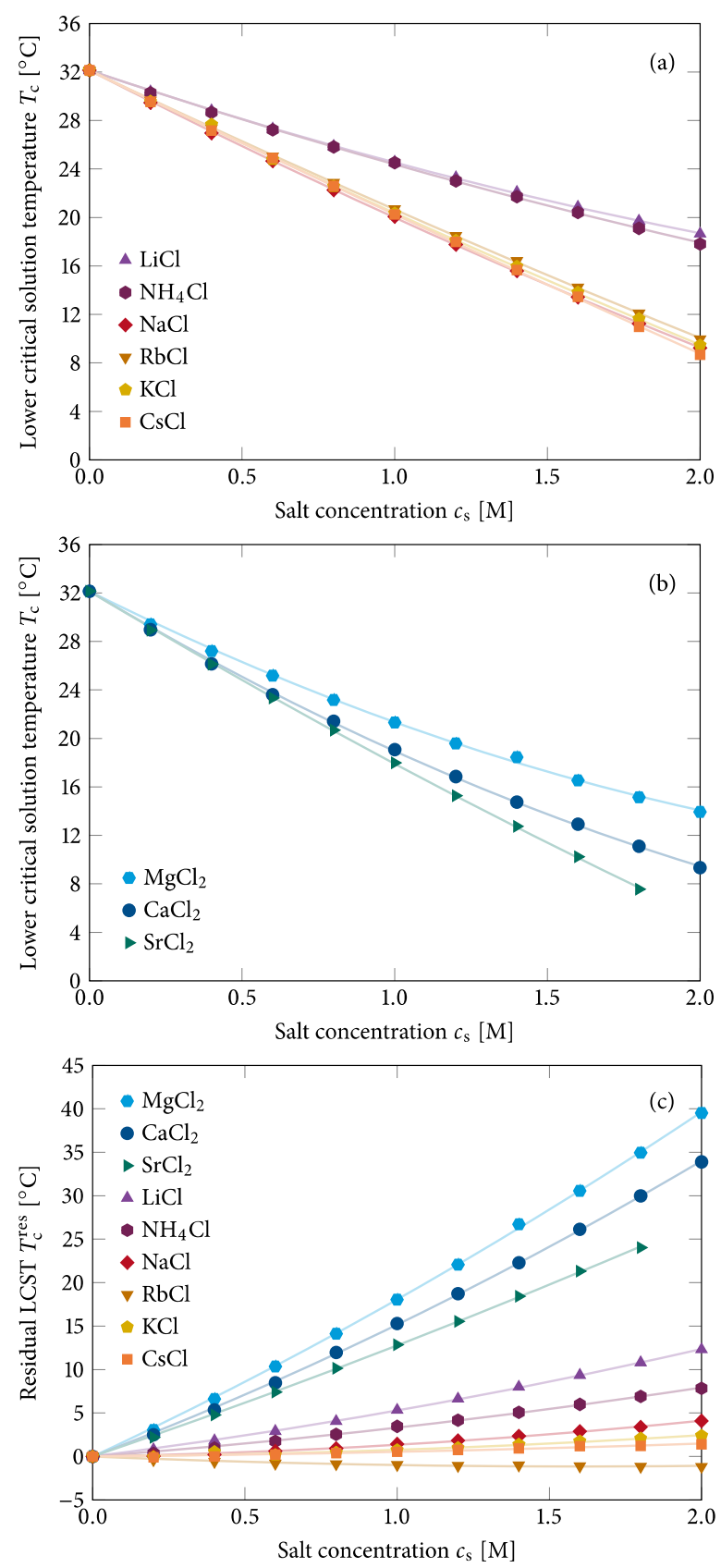

Figure 2. Lower critical solution temperature, $T_{\mathcal{O}}$ for $10 \mathrm{mg} / \mathrm{mL}$ PNIPAM in chloride salt solutions as a function of salt concentration, $c_{s}$, for (a) monovalent and (b) divalent cations. See the Materials and Experimental Techniques sections in the Supporting Information for more details regarding these transition temperature measurements. The symbols are data points, and the lines are fits corresponding to an empirical model (see eqs 1, and S1-S3 in the Supporting Information for details concerning the empirical fitting model for the LCST data). (c) Residual lower critical solution temperature values, $T_{c}^{\text {res }}$, for PNIPAM with all nine chloride salts as a function of salt concentration, $c_{s}$. The symbols represent individual data points, and the lines are fits to the data corresponding to eq 2 (see Table S1 for the fitting parameters for $d$ and $f$ ). Each data point represents an average of six measurements. In each case the error bars, which were calculated from sample standard deviations, are smaller than the size of the data points. Note that the LCST data for $\mathrm{SrCl}_{2}$ are only reported up to its solubility limit, $\sim 1.8 \mathrm{M}$. 
about twice as large as those for the monovalent cations. Nevertheless, the idea that the salting-out behavior should be twice as large on a per molar basis for salts of divalent metal cations does not hold.

The data in Figures $2 \mathrm{a}$ and $2 \mathrm{~b}$ can be fit to an equation of the following form:

$$
T_{c}=T_{0}+a c_{s}+d c_{s}+f c_{s}^{2}
$$

whereby the term $T_{0}$ represents the value of the LCST in the absence of salt. The next term, $a c_{s}$, is a linear salting-out contribution related to the surface tension. By contrast, the third and fourth terms, $d c_{\mathrm{s}}+f c_{\mathrm{s}}{ }^{2}$, represent a salting-in contribution that corresponds to cation binding with the amide oxygen. See eqs S1-S3 in the Supporting Information for more details concerning the empirical model for the LCST. The value of $a c_{\mathrm{s}}$ is known for each salt because it correlates to the value of the surface tension of the air-water interface. ${ }^{8}$ As such, the salting-in contribution from cation binding can be isolated and plotted by subtracting off the $a c_{s}$ term and resetting the initial LCST value to zero (Figure 2c). This residual LCST, $T_{\mathrm{c}}^{\text {res }}$, has the functional form

$$
T_{\mathrm{c}}^{\mathrm{res}}=d c_{\mathrm{s}}+f c_{\mathrm{s}}^{2}
$$

where the coefficients $d$ and $f$ are used for the concentration and concentration squared terms, respectively, with units of ${ }^{\circ} \mathrm{C} / \mathrm{M}$ and ${ }^{\circ} \mathrm{C} / \mathrm{M}^{2}$. The functional form of $T_{\mathrm{c}}^{\text {res }}$ is completely different from a Langmuir isotherm, which arises from saturable macromolecule-ion interactions. Figure $2 c$ generally shows two distinct groups of salts. The strongly hydrated divalent cations $\left(\mathrm{Mg}^{2+}, \mathrm{Ca}^{2+}\right.$, and $\left.\mathrm{Sr}^{2+}\right)$ have a significant salting-in contribution to polymer solubility. By contrast, most monovalent cations $\left(\mathrm{Cs}^{+}, \mathrm{Rb}^{+}, \mathrm{K}^{+}\right.$, and $\left.\mathrm{Na}^{+}\right)$display much weaker influence, although $\mathrm{Li}^{+}$and $\mathrm{NH}_{4}^{+}$show intermediate behavior.

Preferential Binding Coefficients. Next, the interactions of the ions in water with a PNIPAM 20-mer chain were explored by using all-atom MD simulations. The polymer chain that was employed had no end groups as it was connected back onto itself by using periodic boundary conditions. The simulations were performed in solutions of $\mathrm{CaCl}_{2}, \mathrm{LiCl}, \mathrm{NaCl}$, and $\mathrm{CsCl}$ at two concentrations (0.3 and $1.8 \mathrm{~m}$ ). Figure 3 shows the preferential binding coefficients of these salts (cations and anions were treated as indistinguishable in the calculations). Following standard thermodynamic convention, the water is labeled 1 , the polymer chain is labeled 2 , and the ions are labeled 3. The preferential binding coefficient quantifies the excess number of ions in the vicinity of the polymer relative to the statistical number in the bulk solution. When ions partition favorably to the polymer interface, then $\Gamma_{23}>0$. On the other hand, when the ions are depleted, then $\Gamma_{23}<0$.

As can be seen in Figure 3, net depletion from the PNIPAM chain was observed in all cases. The order of $\Gamma_{23}$ between the salts follows the observed LCST order (Figure 2a,b). A 6-fold increase in salt concentration from 0.3 to $1.8 \mathrm{~m}$ led to an $\sim 6-$ fold increase in the absolute value of the preferential binding coefficient for $\mathrm{Na}^{+}$and $\mathrm{Cs}^{+}$. As such, the difference between the polymer-ion and polymer-water affinity remained constant with concentration, ${ }^{39}$ and a linear LCST trend should be expected. Indeed, the chloride salts of these ions led to a nearly linear concentration dependence (Figure 2a). For the strongly hydrated cations $\left(\mathrm{Ca}^{2+}\right.$ and $\left.\mathrm{Li}^{+}\right)$, however, the 6-fold

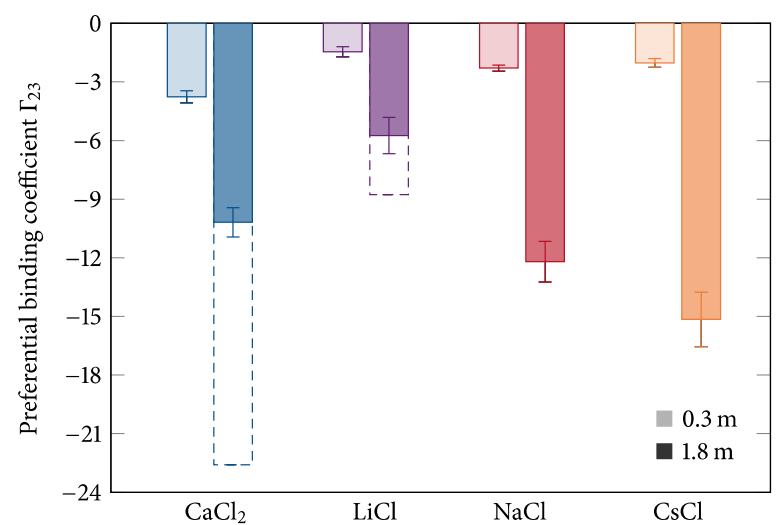

Figure 3. Preferential binding coefficient, $\Gamma_{23}$, corresponding to a PNIPAM 20-mer chain at two salt concentrations with four different salts. The error bars represent the standard deviation of the mean, $\sigma / \sqrt{N}$, using sample standard deviations, $\sigma$, and $N=20(0.3 \mathrm{~m})$ or $N$ $=5(1.8 \mathrm{~m})$ blocks, respectively. The dashed bars depict the preferential binding coefficients that would have been expected if the difference between the polymer-ion and polymer-water affinities had been independent of the salt concentration. Details concerning the MD simulations are provided in the Molecular Dynamics Simulations section in the Supporting Information and in Tables S3 and S4. The Supporting Information also contains more details on the link between the preferential binding coefficients shown in this figure and the LCST values shown in Figure 2. Additionally, distancedependent preferential binding coefficients are provided in Figure S1.

concentration increase yielded a much smaller corresponding change in the preferential binding coefficient. As such, the difference between the polymer-ion and polymer-water affinities was concentration-dependent. Specifically, the salts were less depleted than expected, which should contribute to a smaller drop in the LCST. This should be the origin of the nonlinear trends observed in Figure 2a,b.

Partitioning of Ions to the Macromolecule Surface. The electrolyte structure around the PNIPAM chain was probed in $1.8 \mathrm{~m}$ salt solutions by MD simulations. The normalized proximal radial distribution functions (RDFs) between the polymer backbone and the cations are provided in Figure 4a. In close vicinity to the polymer chain, the $\mathrm{RDF}$ values between the polymer backbone and the cations fell below unity. This is consistent with a net depletion of all ions from the polymer surface. However, the results revealed local accumulation of strongly hydrated cations close to the polymer surface. This accumulation, which can be seen at a distance of $0.75-1.25 \mathrm{~nm}$ from the backbone, came from accumulation around the amide oxygen and terminal methyl groups (Figure S3a). At this distance, the strongly hydrated cations have an intact first hydration shell (see Figure S4). Furthermore, weak but favorable interaction energies were observed between side chain atoms and salt ions (Table S5), in agreement with previous studies. ${ }^{25,35-37,40}$ Namely, net favorable interaction energies were found between the amide oxygen and the cations as well as between the amide nitrogen and chloride and between the two terminal methyl groups and the chloride. Next, Figure $4 \mathrm{~b}$ shows the proximal RDF values between the polymer backbone and the chloride ions. In this case, greater interfacial partitioning for $\mathrm{Cl}^{-}$is observed beyond $0.75 \mathrm{~nm}$ when the countercation was strongly hydrated. Here, too, the peaks in the RDF could be divided into contributions from local accumulation around the amide nitrogen and the terminal 

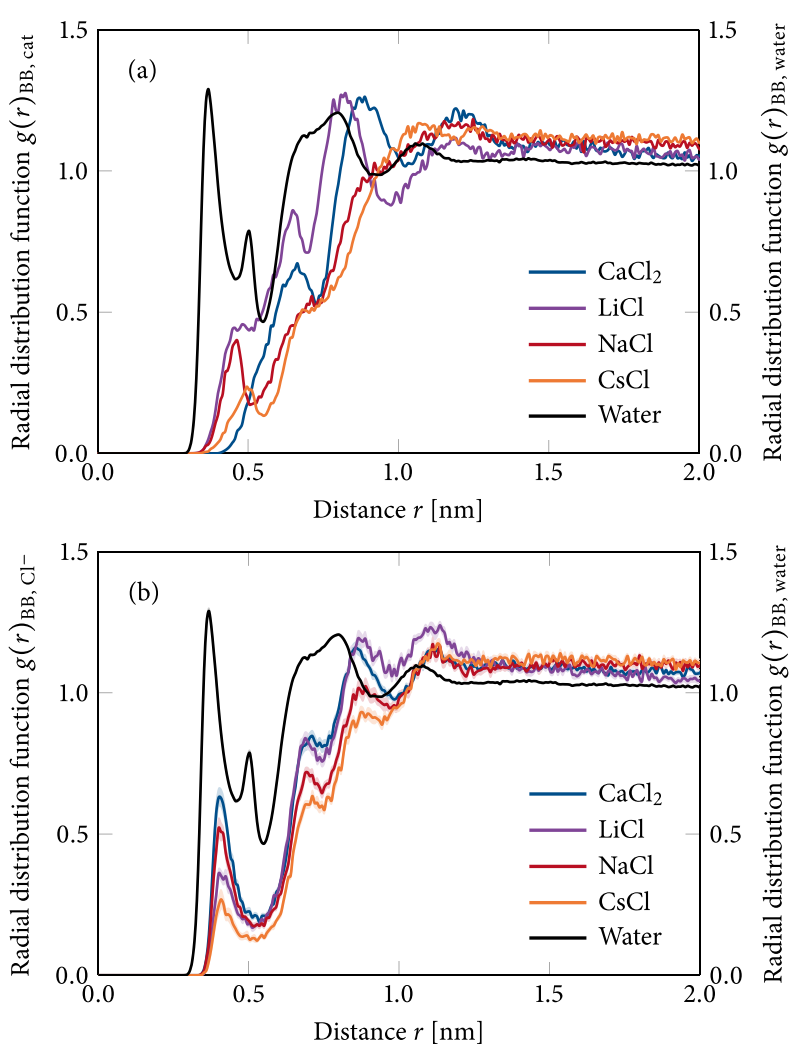

Figure 4. Normalized proximal radial distribution functions, $g(r)$, (a) between the polymer backbone (BB) and the cations (cat) and (b) between the polymer backbone (BB) and the chloride anions as a function of the closest distance, $r$, to the polymer backbone for four salt solutions. The $g(r)$ for the backbone (BB) to water is shown for reference in both figures as black curves (right $y$-axis). The shaded intervals indicate the standard deviation of the mean, $\sigma / \sqrt{N}$, by using sample standard deviations, $\sigma$, and $N=10$ blocks. The error bars are smaller than the thickness of the line when not visible. See Figure S2 for details concerning the cylindrical volume element used for the normalization. Note that the RDF values did not completely converge to 1.0 at longer distances due to the finite volume of the box used in these simulations (i.e., depletion gave rise to an increase in the number of ions in the bulk). Moreover, the stretched chain was approximated by a cylinder for the normalization factor, leading to a deviation from 1.0 for the RDF values at longer distances.

methyl groups (Figure S3b). The presence of both anions and cations in the vicinity of the polymer suggests ion pairing close to the side chains, which is explored in the next section.

Cation-Anion Ion Pairing at the Macromolecular Surface. Figure 5 shows the number density, $\rho(r)$, of solventshared cation-anion pairs (SIPs) normalized to the bulk value, $\rho(r \rightarrow \infty)$, as a function of the closest distance, $r$, between the ion pair and the polymer. Distinct peaks can be seen for the strongly hydrated cations but not for the weakly hydrated ones. Additionally, the number of SIPs was slightly larger in the vicinity of the amide oxygen and the terminal methyl groups compared with the bulk solution (Figure 5 vs Figure S3). Compared with SIPs, the partitioning of contact ion pairs (CIPs) and solvent-separated ion pairs (2SIPs) to the polymer interface was of less importance (Figure S6).

Cations do not typically approach hydrophobic interfaces alone. Ion pairs, however, are neutral species (or reduced in charge when one of the ions is divalent) and can more facilely

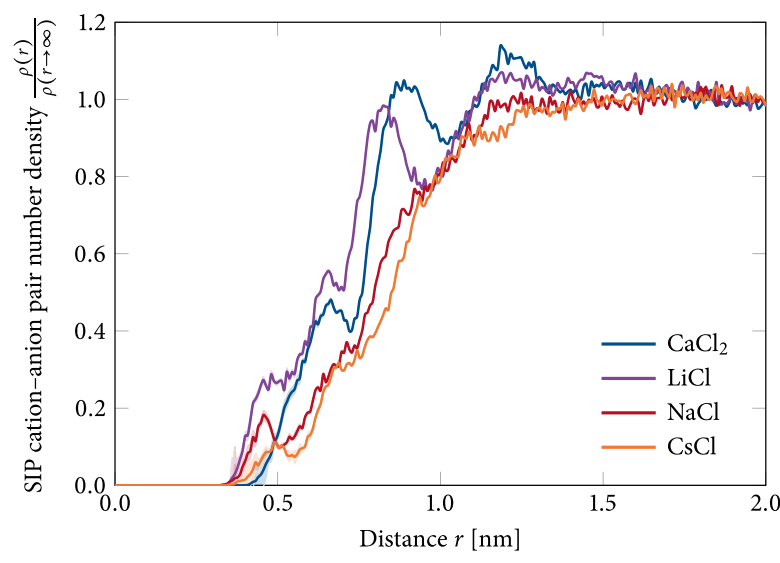

Figure 5. Normalized solvent-shared cation-anion pair (SIP) number density, $\rho(r) / \rho(r \rightarrow \infty)$, as a function of the closest distance, $r$, to the polymer backbone for four chloride salts. See the Supporting Information for details concerning the calculation and Figure S5 for the definition of ion pair types. The shaded intervals indicate the standard deviation of the mean, $\sigma / \sqrt{N}$, by using sample standard deviations, $\sigma$, and $N=10$ blocks and by using propagation of the uncertainty. The error bars are smaller than the thickness of the line when not visible.

approach the surface. In fact, the probability for $\mathrm{Ca}^{2+}$ to partition to the macromolecule surface as an ion pair was very similar to the total partitioning of $\mathrm{Ca}^{2+}$ to the surface. This can be seen from the almost identical spatial probability density maps for $\mathrm{Ca}^{2+}$ alone and for $\mathrm{Ca}^{2+}$ ions in solvent-shared pairs with at least one $\mathrm{Cl}^{-}$(Figure S7).

\section{DISCUSSION AND CONCLUSIONS}

The air/water interface has often been employed as a simple macroscopic proxy for biological interfaces, including the surfaces of proteins. ${ }^{41,42}$ As such, it is instructive to compare the results from the neutral macromolecule/water interface with the air/water interface. In particular, the introduction of ions increases the surface tension of the air/water interface via their depletion from the topmost water layers. ${ }^{24,43}$ If a change in the surface tension was the only contribution to an ion's influence on PNIPAM, then the LCST trends as a function of salt concentration would be directly correlated to how the surface tension changed with increasing concentration for each ion. ${ }^{8}$ As noted above, the anionic Hofmeister series for sodium salts has been shown to be correlated to these values. ${ }^{8}$ By contrast, a similar trend was not seen for the chloride salts examined herein (Figure 2 vs Figure S8). Instead, a substantial residual LCST value remained after subtracting off the surface tension contributions (Figure 2c). Below, the origin of this residual LCST value will be explored.

Ions are typically thought to be repelled from hydrophobic/ aqueous interfaces due to the image charge effect. ${ }^{43}$ In fact, strongly hydrated cations (e.g., $\mathrm{Ca}^{2+}$ ) partition away from the air/water interface, remaining in the bulk solution due to the large favorable interaction energy between the ions and water molecules. This contribution dominates over the unfavorable entropic one caused by the restriction of water molecules in the hydration shells of the ions. This picture, however, is more complicated at the macromolecule/water interface where both hydrophilic and hydrophobic moieties are present. Site-specific interactions can occur at hydrophilic sites (i.e., the amide moieties) for both ions and water. Additionally, even the 
hydrophobic portions of the macromolecule can have dispersion interactions with the solvent. When ions partition toward the macromolecule, weakly favorable interactions between the polymer and the ions (Figures 4 and 5 as well as Table S5) partially compensate for the loss of the favorable interaction energy between the ions and water.

As shown herein, the partitioning of cations to the macromolecular interface is more favorable for strongly hydrated cations (e.g., $\mathrm{Ca}^{2+}$ and $\mathrm{Li}^{+}$) compared to more weakly hydrated ones (e.g., $\mathrm{Na}^{+}$and $\mathrm{Cs}^{+}$) (Figure 4). The number of cations in the vicinity of the macromolecular surface follows the order $\mathrm{Li}^{+}>\mathrm{Ca}^{2+}>\mathrm{Na}^{+}>\mathrm{Cs}^{+}$. The stronger waterion interactions for $\mathrm{Ca}^{2+}$ make it energetically less favorable for it to come to the surface compared to $\mathrm{Li}^{+}$. However, $\mathrm{Ca}^{2+}$ also binds more tightly to the amide oxygen (Table S5). As such, even though there are fewer $\mathrm{Ca}^{2+}$ ions at the interface, they nevertheless have a larger effect on the system compared to $\mathrm{Li}^{+}$, leading to a larger residual LCST value.

Another key consideration for macromolecule solubility is the partitioning of cations paired with chloride counterions as SIPs to the vicinity of the macromolecule (Figure 5). Indeed, it is well-known that ions are often not uniformly distributed in aqueous salt solutions. Instead, cation-anion pairing can be quite abundant at elevated salt concentrations. ${ }^{44,45}$ The results found herein are reminiscent of a recent study showing the surprising presence of two strongly hydrated ions $\left(\mathrm{Mg}^{2+}\right.$ and $\mathrm{SO}_{4}{ }^{2-}$ ) at an air/water interface due to a SIP formation mechanism. ${ }^{46}$ Moreover, the presence of ion pairs near amides also has analogies to ion pair interactions with supramolecular structures. ${ }^{47}$ Indeed, ion pairs are neutral (or reduced in charge for a divalent ion paired with a single monovalent counterion) and thus can approach the macromolecule surface without causing charge separation in the solution. Aided by SIP formation, cations locally accumulate near the amide. This interaction is the basis for the residual LCST values observed in Figure 2c. This contribution is almost large enough in the case of strongly hydrated cations to offset the surface tension contribution. Moreover, the linear term $\left(d c_{\mathrm{s}}\right)$ in eq 2 can putatively be assigned to cation interactions with the amide oxygen, whereas the quadratic term $\left(f c_{s}^{2}\right)$ likely corresponds to ion pair formation in the vicinity of the macromolecule. As can be seen, weakly hydrated cations (e.g., $\mathrm{Na}^{+}$and $\mathrm{Cs}^{+}$) show a very small (or nearly zero) linear term dependence (Table S1) since they have the weakest interactions with the amide oxygen (Table S5). By contrast, the divalent cations as well as $\mathrm{Li}^{+}$have stronger interactions with the amide oxygen and give rise to a steeper slope (Figure 2c). The same strongly hydrated cations (e.g., $\mathrm{Ca}^{2+}$ and $\mathrm{Li}^{+}$) also have a larger nonlinear term due to their enhanced ability to form SIPs with $\mathrm{Cl}^{-}$in the vicinity of the macromolecules (Figure 5). $\mathrm{NH}_{4}{ }^{+}$is an exception due to its directional H-bonding capability. Specifically, its residual salting-in contribution (Figure 2c) is greater than would be expected based on its influence on the air/water surface tension (Table S2). As such, the salting-in contributions described above apply specifically to metal ion chloride salts (Figure 1). As a second example, this mechanism also does not hold for a tetramethylammonium cation (Figure S9). Indeed, this much greasier cation would not be expected to interact specifically with the amide oxygen but rather more generally with the hydrophobic portions of the polymer.

The overall cationic series achieved from macromolecular solubility measurements is $\mathrm{Li}^{+}<\mathrm{NH}_{4}^{+}<\mathrm{Mg}^{2+}<\mathrm{Rb}^{+}<\mathrm{K}^{+}<$ $\mathrm{Cs}^{+}<\mathrm{Na}^{+}<\mathrm{Ca}^{2+}<\mathrm{Sr}^{2+}$, which is significantly different from a direct cationic Hofmeister series. In fact, a direct cationic Hofmeister series can only be observed after subtracting off the surface tension contribution (Figure 2c). The underlying reason for this should be twofold. First, the surface tension contribution leads to a salting-out effect that is mostly dependent on the number of chloride counterions in solution, which differs by a factor of 2 between the monovalent and divalent salts. Second, the ion pairing of cations with chloride helps to give rise to a weak salting-in effect that reinforces the weak direct interactions of the cations with the amide. The ion pairing contribution may follow hard-soft acid-base pairing rules, ${ }^{48}$ the law of matching water affinity, ${ }^{49}$ a partially reversed Hofmeister series, ${ }^{15,23,45,50-52}$ or other pairing rules rather than a direct Hofmeister series. Together, this work shows that strongly hydrated cations, like $\mathrm{CaCl}_{2}$, give rise to LCST phase diagrams wherein the linear salting-out term is nearly offset by both a linear salting-in contribution from very weak binding (i.e., the binding is still on the linear portion of a Langmuir isotherm) and a squared salting-in term for ion pairing. The latter term highlights the fact that the effect of salt ions should not be assumed to be additive.

\section{ASSOCIATED CONTENT}

\section{(5) Supporting Information}

The Supporting Information is available free of charge at https://pubs.acs.org/doi/10.1021/jacs.0c07214.

Materials, transition temperature measurements, empirical model for the lower critical solution temperature of macromolecules, molecular dynamics simulations (system details, simulation details, generation of the elongated chain), preferential binding coefficients, cylindrical volume element for normalization, contributions of heavy side chain atoms to the radial distribution function, hydration numbers, interaction energies between PNIPAM side chains and ions, cation-anion ion pairing structures, probability spatial density maps, surface tension, expected lower critical solution temperatures, and lower critical solution temperatures for other chloride salt solutions (PDF)

\section{AUTHOR INFORMATION}

\section{Corresponding Authors}

Nico F. A. van der Vegt - Eduard-Zintl-Institut für Anorganische und Physikalische Chemie, Technische Universität Darmstadt, D-64287 Darmstadt, Germany; ○ orcid.org/ 0000-0003-2880-6383; Email: vandervegt@cpc.tudarmstadt.de

Sylvie Roke - Laboratory for fundamental BioPhotonics (LBP), Institute of Bioengineering (IBI), and Institute of Materials Science (IMX), School of Engineering (STI), and Lausanne Centre for Ultrafast Science (LACUS), Ecole Polytechnique Féderale de Lausanne (EPFL), CH-1015 Lausanne, Switzerland; 이이.org/0000-0002-6062-7871; Email: sylvie.roke@epfl.ch

Paul S. Cremer - Department of Chemistry and Department of Biochemistry and Molecular Biology, Pennsylvania State University, University Park, Pennsylvania 16802, United States; @i orcid.org/0000-0002-8524-0438; Email: psc11@ psu.edu 


\section{Authors}

Ellen E. Bruce - Eduard-Zintl-Institut für Anorganische und Physikalische Chemie, Technische Universität Darmstadt, D64287 Darmstadt, Germany; ○orcid.org/0000-0001-61934251

Halil I. Okur - Department of Chemistry, and National Nanotechnology Research Center (UNAM), Bilkent University, 06800 Ankara, Turkey; Laboratory for fundamental BioPhotonics (LBP), Institute of Bioengineering (IBI), and Institute of Materials Science (IMX), School of Engineering (STI), and Lausanne Centre for Ultrafast Science (LACUS), Ecole Polytechnique Féderale de Lausanne (EPFL), CH-1015 Lausanne, Switzerland; Department of Chemistry, Pennsylvania State University, University Park, Pennsylvania 16802, United States; ○ orcid.org/0000-0002-2492-1168

Sina Stegmaier - Eduard-Zintl-Institut für Anorganische und Physikalische Chemie, Technische Universität Darmstadt, D64287 Darmstadt, Germany

Chad I. Drexler - Department of Chemistry, Pennsylvania State University, University Park, Pennsylvania 16802, United States; orcid.org/0000-0002-9989-968X

Bradley A. Rogers - Department of Chemistry, Pennsylvania State University, University Park, Pennsylvania 16802, United States

Complete contact information is available at:

https://pubs.acs.org/10.1021/jacs.0c07214

\section{Author Contributions}

E.E.B. and H.I.O. contributed equally to this work.

\section{Notes}

The authors declare no competing financial interest.

\section{ACKNOWLEDGMENTS}

P.S.C. thanks the National Science Foundation (CHE1709735 and CHE-2004050) for support. S.R. acknowledges the Julia Jacobi Foundation and the European Research Council (Grants 240556 and 616305). N.F.A.v.d.V. thanks the LOEWE project iNAPO funded by the Ministry of Higher Education, Research and the ARTS (HMWK) of the state of Hessen. Furthermore, the simulations for this research were conducted on the Lichtenberg high performance computer at TU Darmstadt. The authors also thank Prof. David E. Bergbreiter for providing the PNIPAM.

\section{REFERENCES}

(1) Hofmeister, F. Zur Lehre von Der Wirkung Der Salze. NaunynSchmiedeberg's Arch. Pharmacol. 1888, 24 (4-5), 247-260.

(2) von Hippel, P. H.; Wong, K. Y. Neutral Salts: The Generality of Their Effects on the Stability of Macromolecular Conformations. Science 1964, 145 (3632), 577-580.

(3) Baldwin, R. L. How Hofmeister Ion Interactions Affect Protein Stability. Biophys. J. 1996, 71 (4), 2056-2063.

(4) Cacace, M. G.; Landau, E. M.; Ramsden, J. J. The Hofmeister Series: Salt and Solvent Effects on Interfacial Phenomena. Q. Rev. Biophys. 1997, 30 (3), 241-277.

(5) Record, M. T., Jr.; Zhang, W.; Anderson, C. F. Analysis of Effects of Salts and Uncharged Solutes on Protein and Nucleic Acid Equilibria and Processes: A Practical Guide to Recognizing and Interpreting Polyelectrolyte Effects, Hofmeister Effects, and Osmotic Effects of Salts. Adv. Protein Chem. 1998, 51, 281-353.

(6) Kunz, W.; Henle, J.; Ninham, B. W. 'Zur Lehre von Der Wirkung Der Salze' (About the Science of the Effect of Salts): Franz Hofmeister's Historical Papers. Curr. Opin. Colloid Interface Sci. 2004, 9 (1-2), 19-37.
(7) Kunz, W.; Lo Nostro, P.; Ninham, B. W. The Present State of Affairs with Hofmeister Effects. Curr. Opin. Colloid Interface Sci. 2004, 9 (1-2), 1-18.

(8) Zhang, Y.; Furyk, S.; Bergbreiter, D. E.; Cremer, P. S. Specific Ion Effects on the Water Solubility of Macromolecules: PNIPAM and the Hofmeister Series. J. Am. Chem. Soc. 2005, 127 (41), 1450514510 .

(9) Zhang, Y.; Cremer, P. S. Interactions between Macromolecules and Ions: The Hofmeister Series. Curr. Opin. Chem. Biol. 2006, 10 (6), 658-663.

(10) Pegram, L. M.; Record, M. T., Jr. Hofmeister Salt Effects on Surface Tension Arise from Partitioning of Anions and Cations Between Bulk Water and the Air-Water Interface. J. Phys. Chem. B 2007, 111 (19), 5411-5417.

(11) Cho, Y.; Zhang, Y.; Christensen, T.; Sagle, L. B.; Chilkoti, A.; Cremer, P. S. Effects of Hofmeister Anions on the Phase Transition Temperature of Elastin-Like Polypeptides. J. Phys. Chem. B 2008, 112 (44), 13765-13771.

(12) Pegram, L. M.; Record, M. T., Jr. Thermodynamic Origin of Hofmeister Ion Effects. J. Phys. Chem. B 2008, 112 (31), 9428-9436.

(13) Robinson, D. R.; Jencks, W. P. The Effect of Concentrated Salt Solutions on the Activity Coefficient of Acetyltetraglycine Ethyl Ester. J. Am. Chem. Soc. 1965, 87 (11), 2470-2479.

(14) Lund, M.; Vrbka, L.; Jungwirth, P. Specific Ion Binding to Nonpolar Surface Patches of Proteins. J. Am. Chem. Soc. 2008, 130 (35), 11582-11583.

(15) Hess, B.; van der Vegt, N. F. A. Cation Specific Binding with Protein Surface Charges. Proc. Natl. Acad. Sci. U. S. A. 2009, 106 (32), 13296-13300.

(16) Heyda, J.; Hrobárik, T.; Jungwirth, P. Ion-Specific Interactions between Halides and Basic Amino Acids in Water. J. Phys. Chem. A 2009, 113 (10), 1969-1975.

(17) Lo Nostro, P.; Ninham, B. W. Hofmeister Phenomena: An Update on Ion Specificity in Biology. Chem. Rev. 2012, 112 (4), $2286-2322$

(18) Rembert, K. B.; Paterová, J.; Heyda, J.; Hilty, C.; Jungwirth, P.; Cremer, P. S. Molecular Mechanisms of Ion-Specific Effects on Proteins. J. Am. Chem. Soc. 2012, 134 (24), 10039-10046.

(19) Schwierz, N.; Horinek, D.; Netz, R. R. Anionic and Cationic Hofmeister Effects on Hydrophobic and Hydrophilic Surfaces. Langmuir 2013, 29 (8), 2602-2614.

(20) Xie, W. J.; Gao, Y. Q. A Simple Theory for the Hofmeister Series. J. Phys. Chem. Lett. 2013, 4 (24), 4247-4252.

(21) Hladílková, J.; Heyda, J.; Rembert, K. B.; Okur, H. I.; Kurra, Y.; Liu, W. R.; Hilty, C.; Cremer, P. S.; Jungwirth, P. Effects of EndGroup Termination on Salting-out Constants for Triglycine. J. Phys. Chem. Lett. 2013, 4 (23), 4069-4073.

(22) Jungwirth, P.; Cremer, P. S. Beyond Hofmeister. Nat. Chem. 2014, 6, 261-263.

(23) Schwierz, N.; Horinek, D.; Sivan, U.; Netz, R. R. Reversed Hofmeister Series-The Rule Rather than the Exception. Curr. Opin. Colloid Interface Sci. 2016, 23, 10-18.

(24) Jarvis, N. L.; Scheiman, M. A. Surface Potentials of Aqueous Electrolyte Solutions. J. Phys. Chem. 1968, 72 (1), 74-78.

(25) Okur, H. I.; Hladílková, J.; Rembert, K. B.; Cho, Y.; Heyda, J.; Dzubiella, J.; Cremer, P. S.; Jungwirth, P. Beyond the Hofmeister Series: Ion-Specific Effects on Proteins and Their Biological Functions. J. Phys. Chem. B 2017, 121 (9), 1997-2014.

(26) Murdoch, T. J.; Humphreys, B. A.; Johnson, E. C.; Webber, G. B.; Wanless, E. J. Specific Ion Effects on Thermoresponsive Polymer Brushes: Comparison to Other Architectures. J. Colloid Interface Sci. 2018, 526, 429-450.

(27) Henry, C. L.; Craig, V. S. J. The Link between Ion Specific Bubble Coalescence and Hofmeister Effects Is the Partitioning of Ions within the Interface. Langmuir 2010, 26 (9), 6478-6483.

(28) Nandi, P. K.; Robinson, D. R. The Effects of Salts on the Free Energy of the Peptide Group. J. Am. Chem. Soc. 1972, 94 (4), 12991308 . 
(29) Nandi, P. K.; Robinson, D. R. The Effects of Salts on the Free Energies of Nonpolar Groups in Model Peptides. J. Am. Chem. Soc. 1972, 94 (4), 1308-1315.

(30) von Hippel, P. H.; Peticolas, V.; Schack, L.; Karlson, L. Model Studies on the Effects of Neutral Salts on the Conformational Stability of Biological Macromolecules. I. Ion Binding to Polyacrylamide and Polystyrene Columns. Biochemistry 1973, 12 (7), 1256-1264.

(31) Collins, K. D.; Washabaugh, M. W. The Hofmeister Effect and the Behaviour of Water at Interfaces. Q. Rev. Biophys. 1985, 18 (4), $323-422$.

(32) Leberman, R. M.; Soper, A. M. Effect of High Salt Concentrations on Water Structure. Nature 1995, 378, 364-366.

(33) Parsegian, V. A. Hopes for Hofmeister. Nature 1995, 378, 335336.

(34) Traube, J. The Attraction Pressure. J. Phys. Chem. 1910, 14 (5), $452-470$.

(35) Vrbka, L.; Vondrášek, J.; Jagoda-Cwiklik, B.; Vácha, R.; Jungwirth, P. Quantification and Rationalization of the Higher Affinity of Sodium Over Potassium to Protein Surfaces. Proc. Natl. Acad. Sci. U. S. A. 2006, 103 (42), 15440-15444.

(36) Kherb, J.; Flores, S. C.; Cremer, P. S. Role of Carboxylate Side Chains in the Cation Hofmeister Series. J. Phys. Chem. B 2012, 116 (25), 7389-7397.

(37) Okur, H. I.; Kherb, J.; Cremer, P. S. Cations Bind Only Weakly to Amides in Aqueous Solutions. J. Am. Chem. Soc. 2013, 135 (13), 5062-5067.

(38) Pluhařová, E.; Baer, M. D.; Mundy, C. J.; Schmidt, B.; Jungwirth, P. Aqueous Cation-Amide Binding: Free Energies and IR Spectral Signatures by Ab Initio Molecular Dynamics. J. Phys. Chem. Lett. 2014, 5 (13), 2235-2240.

(39) Heyda, J.; Dzubiella, J. Thermodynamic Description of Hofmeister Effects on the LCST of Thermosensitive Polymers. J. Phys. Chem. B 2014, 118 (37), 10979-10988.

(40) Rembert, K. B.; Okur, H. I.; Hilty, C.; Cremer, P. S. An NH Moiety Is Not Required for Anion Binding to Amides in Aqueous Solution. Langmuir 2015, 31 (11), 3459-3464.

(41) Jungwirth, P.; Tobias, D. J. Specific Ion Effects at the Air/Water Interface. Chem. Rev. 2006, 106 (4), 1259-1281.

(42) Jungwirth, P.; Winter, B. Ions at Aqueous Interfaces: From Water Surface to Hydrated Proteins. Annu. Rev. Phys. Chem. 2008, 59, $343-366$.

(43) Onsager, L.; Samaras, N. N. T. The Surface Tension of DebyeHückel Electrolytes. J. Chem. Phys. 1934, 2 (8), 528-536.

(44) Hawkes, S. J. Salts Are Mostly NOT Ionized. J. Chem. Educ. 1996, 73 (5), 421-423.

(45) van der Vegt, N. F. A.; Haldrup, K.; Roke, S.; Zheng, J.; Lund, M.; Bakker, H. J. Water-Mediated Ion Pairing: Occurrence and Relevance. Chem. Rev. 2016, 116 (13), 7626-7641.

(46) Götte, L.; Parry, K. M.; Hua, W.; Verreault, D.; Allen, H. C.; Tobias, D. J. Solvent-Shared Ion Pairs at the Air-Solution Interface of Magnesium Chloride and Sulfate Solutions Revealed by Sum Frequency Spectroscopy and Molecular Dynamics Simulations. J. Phys. Chem. A 2017, 121 (34), 6450-6459.

(47) He, Q.; Vargas-Zúñiga, G. I.; Kim, S. H.; Kim, S. K.; Sessler, J. L. Macrocycles as Ion Pair Receptors. Chem. Rev. 2019, 119 (17), 9753-9835.

(48) Pearson, R. G. Hard and Soft Acids and Bases. J. Am. Chem. Soc. 1963, 85 (22), 3533-3539.

(49) Collins, K. D. Charge Density-Dependent Strength of Hydration and Biological Structure. Biophys. J. 1997, 72 (1), 65-76.

(50) Zhang, Y.; Cremer, P. S. The Inverse and Direct Hofmeister Series for Lysozyme. Proc. Natl. Acad. Sci. U. S. A. 2009, 106 (36), 15249-15253.

(51) Jordan, J. H.; Gibb, C. L. D.; Wishard, A.; Pham, T.; Gibb, B. C. Ion-Hydrocarbon and/or Ion-Ion Interactions: Direct and Reverse Hofmeister Effects in a Synthetic Host. J. Am. Chem. Soc. 2018, 140 (11), 4092-4099.
(52) Ernst, N. E.; Gibb, B. C. Water Runs Deep. In Supramolecular Chemistry in Water; Kubik, S., Ed.; Wiley-VCH Verlag GmbH \& Co. KGaA: Weinheim, 2019; pp 1-33. 